\title{
Normal state pair nematicity and hidden magnetic order and metal-insulator (fermion-boson)- crossover origin of pseudogap phase of cuprates II
}

\author{
B. Abdullaev ${ }^{1}$, D. B. Abdullaev ${ }^{1}$, C. -H. Park ${ }^{2}$, M. M. Musakhanov ${ }^{3}$ \\ ${ }^{1}$ Institute of Applied Physics, National University of Uzbekistan, Tashkent 100174, Uzbekistan \\ ${ }^{2}$ Research Center for Dielectric and Advanced Matter Physics, Department of Physics, Pusan National University, \\ 30 Jangjeon-dong, Geumjeong-gu, Busan 609-735, Korea \\ ${ }^{3}$ National University of Uzbekistan, Tashkent 100174, Uzbekistan \\ bakhodir.abdullaeff@yandex.ru, cpark@pusan.ac.kr, yousufmm@list.ru
}

PACS 74.72.-h, 74.20.Mn, 74.25.Fy, 74.25.Bt, 74.25.Jb, 74.25.Ha

DOI 10.17586/2220-8054-2017-8-1-59-70

\begin{abstract}
In the present paper II, we will gain an understanding of the nematicity, insulating ground state (IGS), nematicity to stripe phase transition, Fermi pockets evolution, and resistivity temperature upturn, as to be metal - insulator (fermion-boson)- crossover (MIC) phenomena for the pseudogap (PG) region of cuprates. While in the paper I [Abdullaev B., et al. arXiv:cond-mat/0703290], we obtained an understanding of the observed heat conductivity downturn, anomalous Lorentz ratio, insulator resistivity boundary, nonlinear entropy as manifestations of the same MIC. The recently observed nematicity and hidden magnetic order are related to the PG pair intra charge and spin fluctuations. We will try to obtain an answer to the question; why ground state of YBCO is Fermi liquid oscillating and of Bi-2212 is insulating? We will also clarify the physics of the recently observed MIC results of Lalibert et al. [arXiv:1606.04491] and explain the long-discussed transition of the electric charge density from doping to doping +1 dependence at the critical doping. We predict that at the upturns this density should have the temperature dependences $n \sim T^{3} n_{2}$ for $T \rightarrow 0$, where $n_{2}$ is density for dopings close to the critical value. We understood that the upturns before and after the first critical doping have the same nature. We will find understanding of all above mentioned phenomena within PG pair physics.
\end{abstract}

Keywords: high critical temperature superconductivity, cuprate, metal-insulator-crossover, temperature-doping phase diagram, resistivity temperature upturn, insulating ground state, nematicity and stripe phases, Fermi pockets evolution.

Received: 2 August 2016

Revised: 6 August 2016

\section{Introduction}

Information about the normal state raduis of localization for each electron or hole, or its wave function, may play an important role for high temperature $T_{c}$ cuprate superconductors. The size of this raduis may lead to an understanding of the physics of, for example, nematicity and stripe phases. The minimal size for square form nanoregions (NRs) (or of pseudogap (PG) and high $T_{c}$ superconductivity (HTS) pairs), recently measured in the scanning tunneling microscopy (STM) experiments of Gomes et al. [1] and Pan et al. [2] for visualization of the energy gap formation in the cuprate $\mathrm{Bi}_{2} \mathrm{Sr}_{2} \mathrm{CaCu}_{2} \mathrm{O}_{8+\delta}$, has varied between nearly $4.5 a$ and $2.6 a$ of the $a-b$ plane crystal spacing constant $a$, when doping varies between two critical values in the doping-temperature phase diagram. Electric charge analysis of these NRs, made by Abdullaev et al. [3], reveals that at the first critical doping we have one boson inside of each NR, while at the second critical doping, one boson and one fermion. The wide spatial spreading of particle-charge in the underdoped region indicates that the role of the dielectric parent compound of cuprates is unimportant, which leads to universality of the properties of all underdoped copper-oxides with the same $a$. 
The concept of Landau Fermi-liquid quasi-particles (QPs) plays a central role in the understanding of the normal state physics for conventional electric conducting materials [4,5]. Typically, the energies of these QPs depend on momentum and they appear close to the Fermi surface.

For the HTS cuprates (copper-oxides), the normal PG state exhibits highly non-Fermi liquid properties (see, for example, our papers: I [6] and [7], for a comprehensive list of references) and it is unclear whether QPs defined in the momentum space are essential for the PG physics or not. However, as it will be shown in this paper, the real space PG pairs mentioned above and observed in the STM experiment are essential for an understanding of this physics.

In our first paper I [6] we gained an understanding of the following observed MIC phenomena: heat conductivity downturn, anomalous Lorentz ratio, insulator resistivity boundary, and nonlinear entropy. In the present paper (II), we will try to understand of other observed MIC phenomena: IGS, nematicity- and stripe-phases, Fermi pockets evolution, and resistivity temperature upturn. A question can appear about the relation of Fermi pockets evolution to the MIC phenomenon. This question is experimentally studied in detail in the Ref. [8] for $\mathrm{La}_{1.6-x} \mathrm{Nd}_{0.4} \mathrm{Sr}_{x} \mathrm{CuO}_{4}$ copper-oxide. However, the origin of Fermi pockets and what is origin of their evolution with doping has still not been adequately explained in the literature. We will see below that the disconnected Fermi pockets may just be indication of a (fermion) IGS and this state's evolution with doping coincides with these pockets' evolution, while the real origin of the MIC may be bosonic.

The seminal STM experiments of Gomes et al. [1] and Pan et al. [2] on the visualization of the real space PG and HTS NRs, which exhibited an energy gap, together with their electric charge and percolation analysis, made by Abdullaev et al. [3], have deeply extended our understanding of the HTS cuprate physics. The theoretical calculation, shown in the Ref. [3], has further justified the possible existence of single bosons in two-dimensional systems as $a-b$ planes of cuprates. The QPs, PG and HTS pairs-single bosons, have become the fundamental particles in our semi-phenomenological Coulomb single boson and single fermion two liquid model, which positions are formulated in the following list [7].

1. The doping charges, in the form of individual NRs, are embedded in the insulating parent compound of HTS copper oxides. 2. Before the first critical doping $x_{c 1}$ with NR size $\xi_{c o h}=17 \AA$, they are not percolated single bosons. 3. The origin of single bosons is in the anyon bosonization of $2 \mathrm{D}$ fermions. 4 . At the first critical doping $x_{c 1}$, the percolation of single boson NRs and thus HTS appears; there appear also from $x_{c 1}$ doping single fermions, but up to second critical doping, $x_{c 2}$, their NRs do not percolate, thus single fermions between $x_{c 1}$ and $x_{c 2}$ are insulating. 5. The value $x_{c 1}=0.05$ is universal for all copper oxide HTSs, since percolating single boson NRs cover $50 \%$ of the 2D sample area (like connecting squares in a chessboard); the same situation takes place with NRs for fermions at $x_{c 2}$. 6. The normal phase charge conductivity appears from $x_{c 2}$ at $T=0$ or above PG temperature boundary $T=T^{*}$, where the percolation of single fermions appears, while for temperatures between $T_{c}$ and $T^{*}$, there exists (fermion-boson) MIC. 7. The spatially rare charge density object, single boson, with NR size between $\xi_{c o h}=17 \AA$ and $\xi_{c o h}=10 \AA$, which correspond to $x_{c 1}$ and $x_{c 2}$ dopings, has zero total but fluctuating inside of NR spin (this rareness leads also to fluctuating charge inside of NR). 8. The increase of boson spin fluctuations with doping or temperature results in a transition of bosons into fermions, which occurs at PG $T^{*}$ or at $x_{c 2}$. 9. At zero external magnetic field, the HTS is a result of the Bose-Einstein condensate of single bosons. 10. At high external magnetic field strengths, the PG insulating ground state is the result of a plasmon gas consisting of these bosons. We note here that $a \approx 3.8 \AA$.

The majority of these positions are the result of experimental data analysis. Below, when we will discuss positions from this list, we refer to experimental papers, which confirm the validity of these positions, or use the physical arguments for their support. The background of our model, existence of single bosons, has been derived in our microscopic anyon calculation and found its confirmation in the Gomes et al. and Pan et al. [1,2] STM 
experiments. Results of this analysis together with microscopical single boson picture have been combined in the Coulomb two liquid model.

The first two points of these positions have been supported by the experimental paper [9], the authors of which reported on the emergent transition for superconducting fluctuations in the deep antiferromagnetic phase at a remarkably low critical doping, $x_{c}=0.0084$, for ruthenocuprates, $\mathrm{RuSr}_{2}(\mathrm{R}, \mathrm{Ce})_{2} \mathrm{Cu}_{2} \mathrm{O}_{10-\delta}$ with $\mathrm{R}=\mathrm{Gd}, \mathrm{Sm}$, $\mathrm{Nd}$. In this paper, it was claimed that those fluctuations have an intrinsic electronically-inhomogenous nature and provide new support for bosonic models of the superconducting mechanism.

In the introduction section of Ref. [7], we have described a qualitative understanding of the cuprate physics within our Coulomb single boson and single fermion two liquid model. The present paper will be devoted to application of PG pairs for understanding of MIC phenomena listed above. In the Sec. 2 we will give an understanding of the nematicity, IGS, nematicity-stripe phases transition, and Fermi pockets evolution with doping. We explain in detail the physical origin of each phenomenon using the NRs concept. To understand of the resistivity temperature upturn effect, we will use the Sec. 3. In the Sec. 4 we will discuss about the nematicity and hidden magnetic order as manifestations of the intra PG pair charge and spin fluctuations. We argue in this section why these fluctuations may be an indication of the PG pairs. In the Sec. 5, we formulate an interesting question: "Why is the ground state of YBCO an oscillating Fermi liquid and for Bi-2212 is insulating?" This is taken from the experimental analysis and we attempt to answer to it. For interpretation of the recently published experimental MIC results, from the point of view of our Coulomb two liquid model, we will devote the Sec. 6. And we conclude our paper with the Sec. 7 .

\section{Nematicity, IGS, Nematicity-stripe phases transition, and Fermi pockets evolution}

The definition of the intra-unit-cell nematicity order in the copper-oxides is given in the Ref. [10] and it means the breaking of rotational $\mathrm{C}_{4}$ symmetry by the electronic structure within $\mathrm{CuO}_{2}$ unit cell, while the translational symmetry over all cells is conserved. The elemental structure for cuprate's unit cells has square form with $\mathrm{C}_{4}$ rotational symmetry. Authors of Ref. [10] have detected in the STM experiment the prominent electronic structure (charge distribution) along of some sides of this cell, in which the two closest oxygen atoms $\mathrm{O}_{2}$ to the $\mathrm{Cu}$ atom played an important role, for the underdoped $\mathrm{Bi}_{2} \mathrm{Sr}_{2} \mathrm{CaCu}_{2} \mathrm{O}_{8+\delta}$.

However, for underdoped cuprate the area of NR is largest. Thus, a single boson unit charge is spread over the whole NR, which covers several unit cells, giving a very low regional charge density for the NR. The connection of $\mathrm{Cu}$ and $\mathrm{O}$ atoms in the unit cell of parent compound is electrically polarized. It redistributes and fixes a charge inside of the NR. As a result, a pinning of the NR occurs. The pattern of NRs with random pinning of their charges in real space yields the pattern for the electronic nematicity. On the other hand, the soft pinning of NR charges does not destroy the system's translational symmetry.

The pinning of single boson charges in a strong magnetic field (we remember that this magnetic field destroys the HTS and experiment detects IGS (see our paper I)) by the cuprate parent compound is similar to the Wigner crystal of $2 \mathrm{D}$ electrons pinning in a disorder potential and in the same magnetic field. However, this Wigner crystal, under the same external potentials, results in the IGS of electrons (see Refs. [11,12]). Therefore, the plasmon gas of pinned 2D charged single bosons has the IGS. Thus, if the pinned charged bosons are insulating, then the plasmons of this gas is also insulating. The last leads to the IGS of bosonic insulator or to IGS of cuprates (see Refs. [13-17] and point 10. in the above list of our model positions). This can be detected by measuring of this plasmon gas frequency using method described in [11,12]. Then, the bosonic insulator - bosonic metal transition can experimentally be observed at a point for this frequency when it goes to zero. Physically, this transition corresponds to the changing of the charged boson gas elementary excitation spectrum from a plasmon part into a free particle one [6]. 
Authors of Ref. [18] have displayed in their Fig. 8 the schematic evolution of the nematic and stripe charge order phases with doping. They showed that a nematic charge order, with violation of the system's rotational symmetry and small spatial size of this order, dominates at initial dopings of the HTS dome phase diagram. While the stripe phase, with violation of the system's translational symmetry, appears for dopings close to the optimal (critical) level.

The fact that the system's translational symmetry is violated means that a factor, which violates this symmetry, has the same spatial size as $\mathrm{CuO}_{2}$ unit cell. With an increase of doping in the interval between two critical levels and close to the optimal one, the spatial sizes of NRs decrease and single bosons transform into single fermions [3]. Together with insulating single fermions, appearing at the first critical doping (see point 4. of the model positions), the concentration of fermions becomes dominant in the mixture of bosons and fermions. Therefore, for violation of the system's translational symmetry, and thus for a stripe phase, the responsible the NRs occupied by charged single fermions (the real space size of the fermion NR is close to $a$ ).

The schematic evolution of the nematic and stripe charge order phases with doping resembles the bosonic insulator - bosonic metal transition crossover. The temperature-doping boundary of both these evolutions is described by the formula (see Eq. 13 of Ref. [6]):

$$
T_{b I M} \approx 0.368\left[x\left(1-x / x_{c}\right)\right]^{2 / 3} R y,
$$

where $x_{c}$ and $R y$ are the critical doping and Rydberg atomic energy unit, respectively.

Now, it is worthwhile to discuss about Fermi pockets evolution with doping as an additional signature of the MIC. Fermi pockets and stripe phases belong to a very controversial subject of PG cuprate physics. There is a widely accepted belief that there is a relationship between these two objects and furthermore, through Fermi surface reconstruction, stripe phases induce the formation of Fermi pockets (see reviews $[19,20]$ and references therein).

All the problems with understanding of experimental data result from the fact that the QPs, which determine the cuprate physics have so far been considered as fermions. A fermion statistics signature of these QPs has been detected by observation of Fermi pockets, as constituents of a Fermi surface. However, the manifestation of fermions in a parent compound's Brillouin zone starts at low dopings in the form of Fermi arcs. Then, upon evolution with doping, it acquires Fermi pockets, and, finally, after optimal doping, it becomes circle like, as for a homogenous Fermi gas [21].

However, the PG and thus the HTS cuprate physics is closely related with the MIC mechanism discussed in the present paper. As seen from the list of the Coulomb two liquid model positions, the electric current properties, between the first and second critical dopings, are determined by two percolation channels. The first one is conducting channel with percolation of NRs and thus particles inside of each NR; to this channel belongs the bosonic insulator-bosonic metal-fermionic metal transitions.

The second one belongs to the non-percolative, insulating channel. All fermions for dopings between the first and second critical concentrations are from this channel. These charged fermions do not contribute to the PG current properties and physics, therefore, we call them insulating fermions. The experimental information on these fermions cannot be used for clarification of anything in the PG phase physics. Only from the second critical doping, when the percolation of NRs of these fermions together with fermions obtained in the percolative channel starts on, the charged fermions begin to play a role. Everything stated here for insulating fermions is actually for temperatures below the PG boundary $T^{*}$. Percolation starts here from $T^{*}$.

The information about the evolution of Fermi pockets with doping also finds understanding within the insulating fermions. Two critical doping values in the phase diagram temperature-doping of cuprates exactly relate with two maximal and minimal sizes of NRs, which means that distances between particles-fermions varies between these two NR sizes. If we denote the NR size at a given doping $x$ as $\xi_{c o h}(x)$, then two limiting values for the Fermi pocket 
wave vector are approximately determined as $k_{F 1} \approx 2 \pi / \xi_{c o h}\left(x_{c 2}\right)$ and $k_{F 2} \approx 2 \pi / \xi_{c o h}\left(x_{c 1}\right)$. Variation of these two values for the wave vector with doping $x$ is given by formulas $k_{F 1} \approx 2 \pi / \xi_{c o h}\left(x_{c 2}\right)$ and $k_{F 2} \approx 2 \pi / \xi_{c o h}(x)$.

Finally, we note that a Fermi surface in the form of disconnected arcs or pockets is a signature of an insulator rather than a conductor. Because, for the conductor, the wave vector of a charge carrier can be arbitrary inside of a Fermi surface: from zero value up to one of the finite Fermi wave vector [5].

\section{Resistivity temperature upturn}

The validity of the low-temperature (low-T) heat transport Wiedemann - Franz law (WFL):

$$
\kappa /(\sigma T)=L_{0},
$$

where $L_{0}$ is the Lorentz ratio with Sommerfeld's value $L_{0}=\left(\pi^{2} / 3\right)\left(k_{B} / e\right)^{2}$ and $\kappa$ and $\sigma$ are the heat and electric charge conductivities, respectively, has been perfectly proven for many conventional metals [4]. We note that the $L_{0}$ value corresponds to the three dimensional $(3 D)$ case. Simple calculation shows that it can be also applied for the $2 D$ case. An explanation of the WFL for metals was based on the exploition of the simple Drude model for $\kappa$ and $\sigma$ involving Fermi liquid QPs [4]. This model suggests the relation:

$$
\kappa=(1 / 2) c v l
$$

for $2 D$ heat conductivity $\kappa$ (for the $3 D$ case the factor should be $1 / 3$ ) and

$$
\sigma=e^{2} n \tau / m
$$

for the charge conductivity $\sigma$. Here $c, v$ and $l$ in Eq. (3) are the specific heat, mean velocity and mean free penetration length, respectively, and $e, n$ and $\tau$ in Eq. (4) are the charge, $2 D$ density and mean lifetime, respectively, of Fermi liquid QPs with mass $m$. For the low- $T$ limit the $T$ dependence of $\kappa$ is entirely determined by the specific heat $c$. Since the velocity $v$ (as a velocity on the Fermi surface) is a function of $n$ and the length $l \approx v \tau$ is also $T$ independent due to $\tau$ being characterized by scattering of QPs with impurities [5] for these temperatures. For Fermi liquid QPs $c \sim T$ (see Ref. [5]), therefore, $\kappa \sim T$ and this leads to Eq. (2) for WFL of that gas of QPs ( $T$ independence of $\sigma$ is obvious from Eq. (4)).

Hill et al. have reported on the first WFL measurement of electron-doped copper-oxide $\operatorname{Pr}_{2-x} \mathrm{Ce}_{x} \mathrm{CuO}_{4}$ in the pioneer paper [22]. They suppressed the HTS by the strong magnetic field and measured the low- $T$ dependence of the heat conductivity $\kappa$ for the PG QPs of cuprate. The observed $\kappa$ deviated from the normal linear $\kappa \sim T$ behavior into an anomalous $T^{3.6}$ with the decrease of $T$. This behavior, called in the literature as downturn, has also been observed in the other hole-doped copper-oxides (see for references Refs. [6,7]). The measurement of $\kappa$ was performed in these hole-doped copper-oxides likewise after suppression of the HTS by a strong magnetic field.

Smith et al. [23] have suggested by phenomenological treatment that the downturn of $\kappa$ is a result of the decoupling of the heat carrying phonons and electrical charge carriers at low- $T$, while Hill et al. [22] have noted that the downturn is a fundamental intrinsic property of copper oxides. The downturn has been widely accepted as a characteristic of non-Landau Fermi liquid behavior. As the next evidence for the non-Fermi liquid QPs property was recognized the fact that the cuprate Lorentz ratio was significantly larger than Sommerfeld's value [24].

In our preceding paper (Ref. [6]) we have shown that these non-Landau Fermi liquid behaviors of the low- $T$ heat conductivity and Lorentz ratio are results of the MIC property of the cuprate PG regions, in which the IGS originates from a plasmon gas of 2D charged single bosons, pinned by a ferroelectric field of parent compound atoms (see also previous section). In this section, we show that the downturn $T$ dependence carries the important information on the low- $T$ scale of the MIC charge conductivity or resistivity. It will be demonstrated that this scale of the charge conductivity is driven by that of the specific heat. One should note that the physics of the MIC low- $T$ scale of copper-oxides was far from the clear understanding. 
As we argued in Ref. [6], the magnetic field does not affect on the energy of elementary excitations, i.e., plasmons, of gas of charged single bosons. The weak ferroelectric field effect of the cuprate atoms is also negligible for this energy. Therefore, we can use here results for the low- $T$ heat conductivity $\kappa$ and the specific heat $c$, obtained in [6] for the above-mentioned gas of plasmons. Namely, we assume that there exists a some downturn temperature $T_{d}^{\kappa}$ of $\kappa$, which separates the linear $\kappa \sim T$ dependence from the downturn $\kappa \sim T^{4}$ one, in the PG phase of cuprates. Irrespective of the QP nature we consider, one may suppose the validity of WFL, Eq. (2), and Drude model, i.e., Eq. (3) and Eq. (4), for these QPs. However, it is necessarily to replace in these formulas $L_{0} \rightarrow L$, since $L$ has the explicit doping dependence (see Refs. [6, 24]).

On the other hand, the linear dependence $\kappa \sim T$ for $T>T_{d}^{\kappa}$ resembles the case of conventional metals, in which freely penetrating QPs are fermion-like and have $c \sim T$ dependence for the specific heat. Additionally, for low- $T$ and $T>T_{d}^{\kappa}$ we may again apply the above arguments for the $T$ independence of $v$ and $l$ for these QPs as well as conjecture that the temperature dependent $\kappa$ arises from the $T$ dependence of the specific heat $c(T)$.

We introduce the notations $c_{1} \sim T^{4}$ and $c_{2} \sim T$ for specific heats with $T^{4}$ and $T$ dependencies, respectively, and $\sigma_{2}$ for charge conductivity, when $c_{2} \sim T$. Hence, the total specific heat $c$ transits from $c_{1}$ to $c_{2}$ as $T$ increases. The $c \sim T^{4}$ dependence is consistent with the function $\mathcal{S} \sim T^{i}$ with $i>1$ of an entropy (integral $\left.\mathcal{S}=\int_{0}^{T}\left(c / T_{1}\right) d T_{1}\right)$ observed in Ref. [25] for the insulating $\mathrm{YBa}_{2} \mathrm{Cu}_{3} \mathrm{O}_{6+x}$ compound.

In the experimental papers on the WFL of cuprates, authors use the residual value for the charge conductivity $\sigma_{2}$, which is the intercept of $\sigma_{2}$, measured for $T \geq T_{d}^{\kappa}$ range, by extrapolation to the $T \rightarrow 0$ limit. It was previously shown (see Refs. [5]) that the typical low- $T \sigma_{2}$ is nearly constant, which is in accordance with the above assumption regarding the scattering of freely penetrating QPs with impurities. Therefore, one assumes $\sigma=\sigma_{2} \approx$ const in WFL, Eq. (2), in the experimental measurements in this subject. We substitute the expressions for $\kappa$, Eq. (3), and $\sigma_{2}$, Eq. (4), in the left hand side of Eq. (2). Then recalling that $\kappa$ is expressed by the total specific heat $c$, one obtains the formula

$$
\kappa /\left(\sigma_{2} T\right)=c L / c_{2}
$$

for the measured WFL of cuprates, where the constant $L$ is defined by the expression $L=c_{2} m v^{2} /\left(2 e^{2} n T\right)$. It is clear that $L$ is determined for the $T \geq T_{d}^{\kappa}$ temperatures, for which the "metallic" QPs are well defined. We cannot calculate the numerical value of $L$. Since numerical data of empirical quantities, through which it is expressed, are unknown. However, from the paper [24] of Proust et al. we know that $L$ is constant for fixed doping. Therefore, Eq. (5) demonstrates that for fixed doping, the $T$ dependence of WFL is provided by $c / c_{2}$ factor and vice versa, the observed data on WFL determine the $T$ dependent $c / c_{2}$.

Let us suppose that the WFL for copper-oxides is temperature, $T$, independent as for conventional metals and defined by the relation $\kappa /(\sigma T)=L$, where $\sigma$ varies now in the whole interval of $T$. Multiplying and dividing the left hand side of this relation by $\sigma_{2}$ and using Eq. (5) we obtain the expression

$$
\sigma=c \sigma_{2} / c_{2}
$$

which is the main result of the present section. Then, Eq. (6) has two asymptotic limits: $\sigma=\sigma_{2}$ for $T>T_{d}^{\kappa}$, when $c=c_{2}$, and $\sigma \sim T^{3} \sigma_{2}$ for $T<T_{d}^{\kappa}$ and $T \rightarrow 0$, when $c \sim T^{4}$. While for the first limit, the charge conductivity $\sigma$ equals that of the conductor $\sigma_{2}$ (since $\sigma_{2}=$ const $\neq 0$ ). For the second limit, due to vanishing of $\sigma$ at $T \rightarrow 0$, we have the conductor to insulator transition or the MIC. The important thing is the insulator charge conductivity has the $\sigma \sim T^{3}$ dependence. This $T$ dependence for $\sigma$ may be used in the experiment for the indication of the insulating state.

We may clarify the origin of the MIC crossover. For this we extend the Drude formula, Eq. (4), for the charge conductivity $\sigma$ to the all considered temperatures (including the $T<T_{d}^{\kappa}$ interval) and define the concentration of charge carriers $n$, which corresponds to the $\sigma$. Let the "metallic" conductivity $\sigma_{2}$ be defined by Eq. (4) itself with the charge concentration $n_{2}$. Substituting both the Drude formulas for $\sigma$ and $\sigma_{2}$ in the Eq. (6) we obtain 


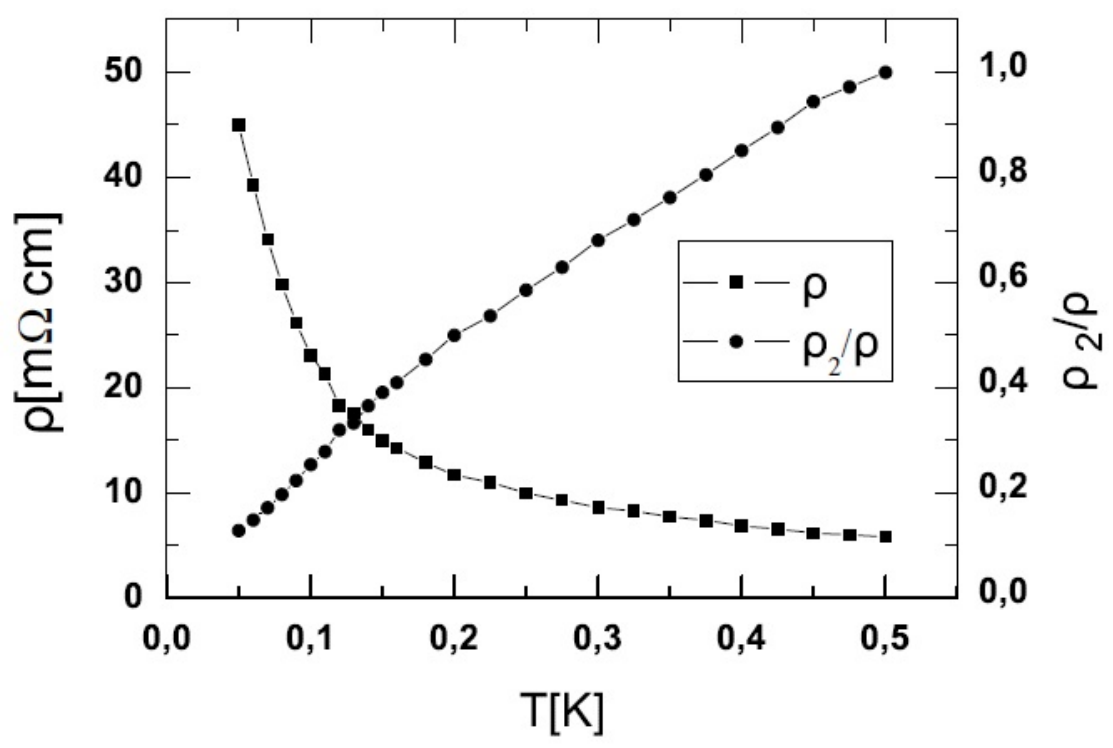

FIG. 1. The low- $T$ dependence of a resistivity $\rho$ for $\mathrm{La}_{2-x} \mathrm{Sr}_{x} \mathrm{CuO}_{4+\delta}$ copper-oxide with $x=$ 0.06 (from Fig. 3 (a) of Ref. [26]) and constructed on the base of this $\rho$ our ratio $\rho_{2} / \rho$. It was assumed that metallic $\rho_{2}=\rho(T=0.5 \mathrm{~K})$

$n=c n_{2} / c_{2}$. In the last equation, we have implied the reasonable argument that other parameters of the gas of QPS (except the concentration $n$ ) should not change in the MIC. From this expression for $n$, we see that $n=n_{2}$ for $T \geq T_{d}^{\kappa}$, when all charge carriers are the freely penetrating QPs, and $n \sim T^{3} n_{2} \rightarrow 0$ for $T<T_{d}^{\kappa}$ and $T \rightarrow 0$. The second limit of $n$ corresponds to vanishing of QPs. The decrease in the density of the conducting carriers is a reason for the MIC of cuprates. It is also the origin of the decoupling of the heat-carrying thermal phonons with these charge carriers [23].

The dependence for the concentration of carries, $n \sim T^{3} n_{2}$, as a function of temperature, $T$, and at fixed doping, $x$, can be experimentally measured for any copper-oxide by using the method, described, for example, in the Ref. [27].

In Fig. 1, we plot the low- $T$ dependence of a resistivity $(\rho=1 / \sigma)$ taken from Fig. 3(a) of Ref. [26] forLa $\mathrm{La}_{2-x} \mathrm{Sr}_{x} \mathrm{CuO}_{4+\delta}$ copper-oxide with $x=0.06$. It is also shown in this figure the ratio $\rho_{2} / \rho=\sigma / \sigma_{2}$, where we assumed that $\rho_{2}=\rho(T=0.5 \mathrm{~K})$. The observed in [26] result for $\rho$ corresponds to the insulating normal state of the compound obtained by suppression of HTS by a strong magnetic field. As seen from Fig. $1, \rho_{2} / \rho$ line has the downturn behavior below $T=0.5 \mathrm{~K}$ and an obvious non-linear dependence for $T \leq 0.15 \mathrm{~K}$. This downturn relates to an MIC. However, since the slope of the depicted $\rho(T)$ is negative at $T=0.5 \mathrm{~K}$, the $\rho_{2} / \rho=1$ assumption is approximate, and the copper-oxide is still in the crossover state at this $T$. The downturn region of $\rho_{2} / \rho$ extends for higher temperatures.

In Fig. 2, we compare in the semi-log scale the low- $T$ dependence of $\rho(T)$ measured for purely insulating state $x=0.05$ (without magnetic field) of the same copper-oxide (the insert of Fig. 3 (a) in Ref. [26]) with our empirically found $(a T)^{-3}$ function, where $a=0.464 \mathrm{~K}^{-1}$. As is evident from this figure, our function satisfactorily fits the non-linear part of the observed $\rho(T)$ for the lowest temperatures. This non-linear $T$ dependence of $\rho(T)$ is also seen in Fig. 1 for lowest $T$. One may assume both insulating states have the same origin and for both, the $T^{-3}$ dependence of $\rho(T)$ is valid.

At the end of this section, we note that the IGS of copper-oxides, the origin of which is the plasmon gas of 2D charged single bosons, results in the resistivity temperature upturn at the low- $T$ limit as at strong magnetic field and $x>x_{c 1}$, when the HTS is suppressed by this field, so and for purely insulating state with $x<x_{c 1}$ and 


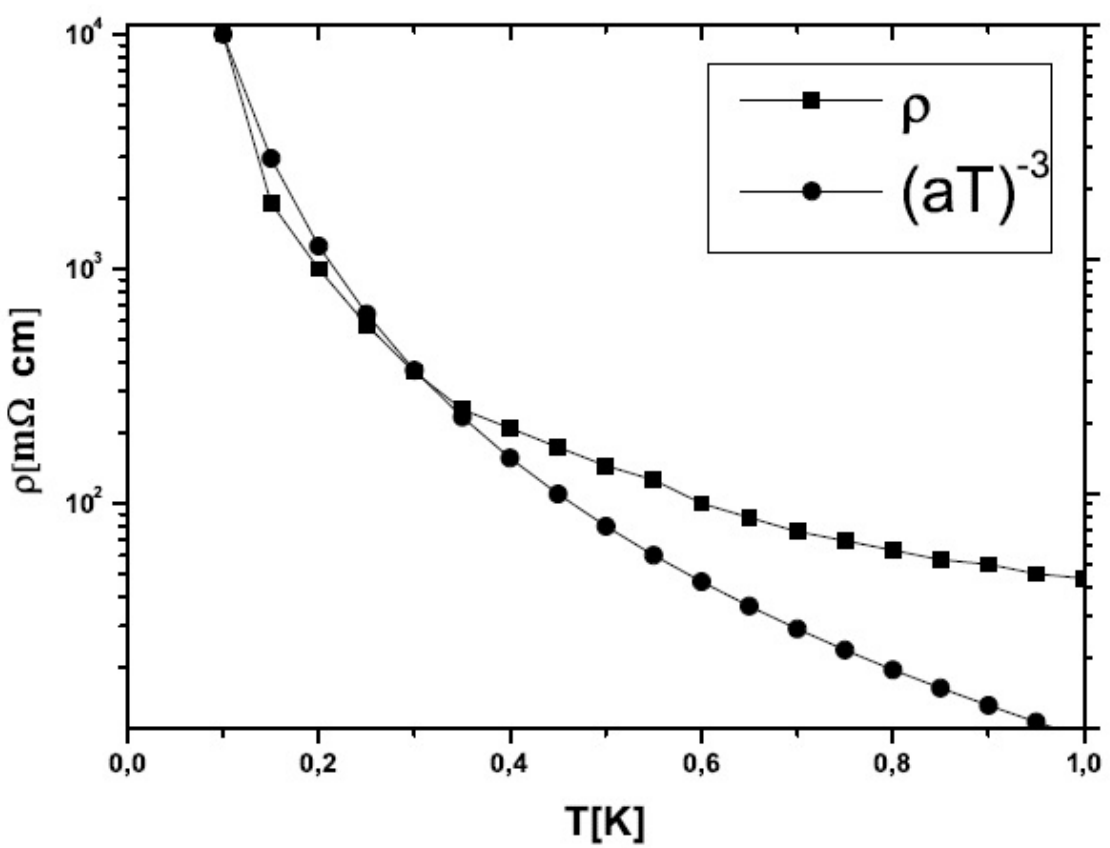

FIG. 2. The semi-log scale of the low- $T$ dependence of $\rho$ for $\mathrm{La}_{2-x} \mathrm{Sr}_{x} \mathrm{CuO}_{4+\delta}$ copper-oxide with $x=0.05$ (measured without a magnetic field and below the first critical doping) (the insert of Fig. 3 (a) of Ref. [26]) and our empirically fitted function, $-3 \log (a T)$, with $a=0.464 K^{-1}$. It is seen that our function satisfactorily represents the non-linear part of the observed $\rho$ at lowest $T$

without field. This is in accordance with point 10 of the Coulomb two liquid model positions and confirms the point 1, which means that, even though at $x<x_{c 1}$ the electric charges and their NRs are not percolating, due to plasmon origin of the IGS, the resistivity displays the upturn behavior. In principle, the last argument can also be applied for insulating fermions for dopings in the interval $x_{c 1} \leq x \leq x_{c 2}$. It seems that they should give a similar upturn in the resistivity. However, the weight of their concentration with respect to one of single bosons for the same dopings is not high.

\section{Nematicity and hidden magnetic order as intra PG pair charge and spin fluctuations}

First observed in $\mathrm{YBa}_{2} \mathrm{Cu}_{3} \mathrm{O}_{6+x}$ (Y123) compound, using polarized elastic neutron diffraction [28], the hidden magnetic order has been observed in three other copper-oxide families: $\mathrm{HgBa}_{2} \mathrm{CuO}_{4+\delta}(\mathrm{Hg} 1201)$ [29, 30], $\mathrm{La}_{2-x} \mathrm{Sr}_{x} \mathrm{CuO}_{4}$ (La214) [31] and $\mathrm{Bi}_{2} \mathrm{Sr}_{2} \mathrm{CaCu}_{2} \mathrm{O}_{8+\delta}$ ( $\mathrm{Bi}-2212$ ) [32] (see for references also [33]). Since the size of detection was a few structural cells, it was called the intra-unit-cell (IUC) hidden magnetic order.

These interesting experiments have revealed the existence of IUC objects in the PG phase with fluctuating spin components inside, which exactly cancel each other, so that the total spin of the every object was zero. Despite the authors of Ref. [28] having interpreted the physics of both, total and intra, spins either by invoking of a pair of oppositely flowing intra structural cell charge loop-currents or of staggered spins in the same cell, the role of these objects in the physics of copper-oxides was not understood [34].

This role becomes unambiguously clear, if we connect these objects with the visualization of NRs which exhibit the energy gap in the STM experiment [1]. Since, as NRs, they exist in the PG region and disappear in the PG temperature boundary $T^{*}$ [28]. There is no doubt that their evolution with temperature will be the same as for NRs. While their evolution with doping (see Refs. [28-30]) qualitatively coincides with that of the NRs, described in [3]. However, minimal size NRs are single bosons, therefore, the IUC hidden magnetic order objects are also 
single bosons and PG and HTS pairs. On the other hand, the spin fluctuation inside of the single boson was introduced in our original papers on the single boson mechanism of HTS [35,36]. Furthermore, the increase of this fluctuation with temperature below PG boundary $T^{*}$ and doping below the second critical $x_{c 2}$ and transformation of these single bosons into single fermions in $T^{*}$ and $x_{c 2}$ was physical origin of $T^{*}$ and $x_{c 2}$.

The spatially intra rare charge density of each single boson allows one to understand the nature of the intra unit cell electronic nematicity, observed recently in the STM experiment [10], as the intra PG pair dynamic charge fluctuation. The strong ferrielectric crystal field of the parent compound forms an atomic scale charge distribution within an individual NR (see for details the Sec. 2).

The evolution of the dynamic charge fluctuation with doping resembles that of the IUC hidden magnetic order [21] (Ref. [21] contains a very thorough up-to-date list of references on the subject of IUC spin and charge fluctuations).

\section{Why ground state of $\mathrm{YBCO}$ is Fermi liquid oscillating and of Bi-2212 is insulating?}

The Fermi liquid like behavior of conducting QPs have been observed through the measurement of magnetoresistance oscillations at high magnetic fields in the underdome region of the temperature-doping phase diagram. The authors of these quantum oscillation experiments have succeeded in measuring a Fermi surface, which had a form of Fermi pockets. This effect was found for the YBCO family of cuprates for dopings up to the optimal level (see the review of Ref. $[19,20]$ for references).

However, the quantum oscillations were not found in other copper-oxides, whose ground states were insulating in the same magnetic fields (see Refs. [13-17]). There appears the question: Why the ground state of some copper-oxide compounds is Fermi liquid oscillating and of other cuprates is insulating and can this discrepancy be understood within the framework of a single model?

If we look on the Table 1, we see that the critical doping for the YBCO cuprate is $x_{c}=0.19$, while for the $\mathrm{Bi}-2212$ one is $x_{c}=0.28$. The lowest critical $x_{c}$ provides the percolation for the single fermion NRs at the lower dopings, close to $x_{c}$. Thus the YBCO cuprate has the Fermi liquid oscillating ground state. While for Bi-2212, i.e., $\mathrm{Bi}_{2} \mathrm{Sr}_{2} \mathrm{CaCu}_{2} \mathrm{O}_{8+\delta}$ HTS compound, the single fermion NRs are not percolating for almost all $x$ between $x_{c 1}$ and $x_{c 2}$. Therefore, the ground state of $\mathrm{Bi}-2212$ material in a strong magnetic field is insulating (see Ref. [37]). However, it is worthwhile to note here that the cuprate $L S C O$ with low critical doping $x_{c}=0.18$ (see Table 1) has, for unknown reasons to us, an insulating ground state (see Refs. [13-17]).

\section{Interpretation of experimental MIC results 2016}

The resistivity of cuprates at low temperatures and in high magnetic fields revealed the appearance of an insulator-like upturn in its temperature dependence [13-17], which we already discussed in Sec. 3. This upturn (MIC) behavior is still considered puzzle in the literature.

Using high-field resistivity measurements on $\mathrm{La}_{2-x} \mathrm{Sr}_{x} \mathrm{CuO}_{4}$, Lalibert $\mathrm{F}$. et al. [27] have tried to experimentally show that this MIC upturn property is due to a drop in carrier density $n$ associated with the onset of the PG phase at the critical doping $x_{c}$. Namely, authors of Ref. [27] proposed that the upturns are quantitatively consistent with a drop from $n=1+x$ above $x_{c}$ to $n=x$ below $x_{c}$. Based on that idea, the explanations of the upturns for other copper-oxides are also given in [27]. Here, we note that this transition in carrier density $n$ dependence is the long term discussed in a literature issue (see [27] for references).

First, we start with mentioning (see Sec. 2) that all electric current properties of cuprates should be considered within the conducting percolation channel. Therefore, the resistivity temperature upturn is a result of this channel, more exactly, as we demonstrated in Sec. 3, of the plasmon gas of 2D charged single bosons. No charged single fermions below the critical doping $x_{c}$ play a role. Their NRs are not percolating, thus these fermions are insulating. 
TABLE 1. Carrier density as function of normalized to $x_{c}$ doping for $\mathrm{LSCO}=\mathrm{La}_{2-x} \mathrm{Sr}_{x} \mathrm{CuO}_{4}$, $\mathrm{YBCO}=\mathrm{YBa}_{2} \mathrm{Cu}_{3} \mathrm{O}_{y}$ and $\mathrm{Nd}-\mathrm{LSCO}=\mathrm{La}_{1.6-x} \mathrm{Nd}_{0.4} \mathrm{Sr}_{x} \mathrm{CuO}_{4}$, taken from Ref. [27], and for fermion carrier density for $\mathrm{Bi}-2212=\mathrm{Bi}_{2} \mathrm{Sr}_{2} \mathrm{CaCu}_{2} \mathrm{O}_{8+\delta}$, taken from Ref. [3]. For the comparison, in the brackets we showed numerical values for $\mathrm{Bi}-2212$, if the function was measured in the experiment

\begin{tabular}{|c|c|c|c|c|c|}
\hline$x / x_{c}$ & 0.2 & 0.4 & 0.6 & 0.8 & 1.0 \\
\hline LSCO,$x_{c}=0.18$ & & & & & \\
$n\left(x / x_{c}\right)$ & 0.04 & 0.07 & 0.1 & 0.14 & 1.18 \\
\hline YBCO, $x_{c}=0.19$ & & & & & \\
$n\left(x / x_{c}\right)$ & 0.04 & 0.08 & 0.11 & 0.15 & $1.4 \pm 0.4$ \\
\hline $\begin{array}{c}\text { Nd-LSCO, } x_{c}=0.235 \\
n\left(x / x_{c}\right)\end{array}$ & 0.05 & 0.09 & 0.14 & 0.188 & $1.3 \pm 0.4$ \\
\hline $\mathrm{Bi}-2212, x_{c}=0.28$ & $(0.06)$ & $(0.112)$ & $(0.168)$ & $(0.224)$ & $(1.3 \pm 0.4)$ \\
$f(x)=n\left(x / x_{c}\right)-1$ & -0.103 & 0.170 & 0.341 & 0.524 & 0.939 \\
\hline
\end{tabular}

Secondly, one needs to make clear the definition of a carrier density. Because it does not simply indicate the fraction of charge $x$ per $\mathrm{Cu}$ atom. As we already discussed in previous sections, only the NR, i.e. the PG pair, carries the charge $n$ in the normal state. And for doping $x$ between critical $x_{c 1}$ and $x_{c 2}$ we have $n=1+f(x)$, where the charge of boson is 1 and the charge of fermion is $f(x)$. Here, the function $f(x)$ is phenomenological and taken from the STM experimental data for NRs (see Table 1 for Bi-2212). Therefore, the fraction $f(x)$ indicates the charge of fermion inside the PG pair. At the approach of $x$ to $x_{c 2}$, or for PG pair size approaching that of $2 a$, the charge of fermion tends to 1 and thus each $\mathrm{Cu}$ atom contains one doped fermion-hole. This is reason why at critical doping $x_{c}$ in Ref. [27] the charge density adds 1 or becomes $n=1+x$.

Lalibert F. et al. claim in the Ref. [27] that the transition $n=1+x$ into $n=x$ for the carrier density $n$ has a sharp, like in a second order phase transition, form and it occurs exactly at the critical doping $x_{c}$. However, the original Refs. [13-17] result on the upturns was MIC, which means a soft gradual transition, and it occurred according to [13-17] at "near optimal doping", i.e., below critical doping $x_{c}$. Furthermore, Boebinger et al. demonstrated in the Ref. [17] that this MIC temperature-doping boundary resembles the PG boundary $T^{*}$ but located below $T^{*}$. This MIC boundary approximately describes by Eq. (1) and corresponds to bosonic insulatorbosonic metal transition boundary, as was discussed in our Ref. [6], while the total PG phase MIC corresponds to bosonic insulator-bosonic metal-fermionic metal transitions [7]. The last transition occurs at $T^{*}$.

One needs to note at the end of this section that the fraction of dopings corresponding to single bosons is hidden from the Luttinger sum rule investigations of copper oxides. This rule investigates only fermions, however, there is a variety of publications, which supports or violates the Luttinger sum rule for these materials. Refs. [38, 39], for example, violates this rule.

\section{Conclusion}

In conclusion, we have found an understanding for the nematicity, IGS, nematicity to stripe phase transition, Fermi pockets evolution, and resistivity temperature upturn, as to be MIC phenomena for the PG region of cuprates. While in our initial paper, we have found an understanding of the observed heat conductivity downturn, anomalous Lorentz ratio, insulator resistivity boundary, nonlinear entropy as manifestation of the same MIC. The observed nematicity and hidden magnetic order have been related to the PG pair intra charge and spin fluctuations. Probably, we could understand the answer on the question: Why ground state of YBCO is Fermi liquid oscillating and of 
Bi-2212 is insulating? We have also analyzed the physics of the recently observed MIC result of Lalibert et al. [27] and explained the long term discussed in the literature transition of the electric charge density from doping to doping +1 dependence at the critical doping. We predict that at the upturns this density should have a temperature dependence of $n \sim T^{3} n_{2}$ for $T \rightarrow 0$, where $n_{2}$ is density for dopings close to the critical level. We understood that the upturns before and above first critical doping have the same nature. We found understanding of all above mentioned phenomena within the PG pair physics.

\section{Acknowledgements}

Authors B. Abdullaev, D. B. Abdullaev and M. M. Musakhanov acknowledge the support of the research by the Volkswagen Foundation of Germany and C. -H. Park by the National Research Foundation of Korea (NRF) grant funded by the Korean Government (2015M3D1A1070639).

\section{References}

[1] Gomes K. K. et al. Visualizing pair formation on the atomic scale in the high- $T_{c}$ superconductor $\mathrm{Bi}_{2} \mathrm{Sr}_{2} \mathrm{CaCu}_{2} \mathrm{O}_{8}+\delta \cdot N a t u r e, 2007,447$, P. 569-572.

[2] Pan S. H. et al. Microscopic electronic inhomogeneity in the high- $T_{c}$ superconductor $\mathrm{Bi}_{2} \mathrm{Sr}_{2} \mathrm{CaCu}_{2} \mathrm{O}_{8+x}$. Nature, 2001,413 , P. $282-285$.

[3] Abdullaev B., Park C.-H., Musakhanov M. M. Anyon bosonization of 2D fermions and single boson phase diagram implied from experiment on visualizing pair formation in superconductor $\mathrm{Bi}_{2} \mathrm{Sr}_{2} \mathrm{CaCu}_{2} \mathrm{O}_{8+\delta}$. Physica C, 2011, 471, P. 486-491.

[4] Ashcroft N. W., Mermin N. D. Solid State Physics. Philadelphia, HRW, 1976.

[5] Abrikosov A. A. Fundamentals of the Theory of Metals. Amsterdam, Elsevier Science, 1988.

[6] Abdullaev B., Park C.-H., Park K. -S., Kang I. -J. Metal-insulator (fermion-boson)-crossover origin of pseudogap phase of cuprates I: anomalous heat conductivity, insulator resistivity boundary, nonlinear entropy. ArXiv e-prints, 2007, arXiv:cond-mat/0703290, 8 pp.

[7] Abdullaev B., Abdullaev D. B. Park C.-H., Musakhanov M. M. Intra pseudogap- and superconductivy-pair spin and charge fluctuations and underdome metal-insulator (fermion-boson)-crossover phenomena as keystones of cuprate physics. Nanosystems: Phys. Chem. Math., 2015 , 6, P. 803-824.

[8] Collignon C. et al. Fermi-surface transformation across the pseudogap critical point of the cuprate superconductor $\mathrm{La}_{1.6}-{ }_{x} \mathrm{Nd}_{0.4} \mathrm{Sr}_{x} \mathrm{CuO}_{4}$. ArXiv e-prints, 2016, arXiv: 1607.05693, 11 pp.

[9] Mclaughlin A.C., Attfield J.P. Emergent Transition for Superconducting Fluctuations in Antiferromagnetic Ruthenocuprates. Phys. Rev. $B, 2014,90$, P. R220509.

[10] Lawler M. J. et al. Intra-unit-cell electronic nematicity of the high- $T_{c}$ copper- oxide pseudogap states. Nature, 2010 , 466, P. 347-351.

[11] Drichko I. L. et al. Melting of Wigner crystal in high-mobility n-GaAs/AlGaAs heterostructures at filling factors $0.18>\nu>0.125$ : Acoustic studies. ArXiv e-prints (2016), ArXiv: 1607.01918, 6 pp.

[12] Shayegan M. Flatland electrons in high magnetic fields. In High Magnetic Fields: Science and Technology, Vol. 3, edited by F. Herlach and N. Miura, Singapore, World Scientific Co, 2006, P. 31-60, ArXiv e-prints, 2005, ArXiv: cond-mat/0505520.

[13] Ando Y. et al. Logarithmic Divergence of both In-Plane and Out-of-Plane Normal-State Resistivities of Superconducting $\mathrm{La}_{2-x} \mathrm{Sr}_{x} \mathrm{CuO}_{4}$ in the Zero-Temperature Limit. Phys. Rev. Lett., 1995, 75, P. 4662-4665.

[14] Fournier P. et al. Insulator-Metal Crossover near Optimal Doping in $\operatorname{Pr}_{2-x} \mathrm{Ce}_{x} \mathrm{CuO}_{4}$ : Anomalous Normal-State Low Temperature Resistivity. Phys. Rev. Lett., 1998, 81, P. 4720-4723.

[15] Ono S. et al. Metal-to-Insulator Crossover in the Low-Temperature Normal State of $\mathrm{Bi}_{2} \mathrm{Sr}_{2}-{ }_{x} \mathrm{La}_{x} \mathrm{CuO}_{6+\delta}$. Phys. Rev. Lett., 2000, 85, P. 638-641.

[16] Ando Y. et al. Supporting evidence of the unusual insulating behavior in the low-temperature normal-state resistivity of underdoped $\mathrm{La}_{2-x} \mathrm{Sr}_{x} \mathrm{CuO}_{4}$. J. Low Temp. Phys., 1996, 105, P. 867-875.

[17] Boebinger G. S. et al. Insulator-to-Metal Crossover in the Normal State of $\mathrm{La}_{2-x} \mathrm{Sr}_{x} \mathrm{CuO}_{4}$ Near Optimum Doping. Phys. Rev. Lett., 1996, 77, P. 5417-5420.

[18] Caprara S. et al. Signatures of nematic quantum critical fluctuations in the Raman spectra of lightly doped cuprates. Phys. Rev. B, 2015, 91, P. 205115.

[19] Vojta M. Lattice symmetry breaking in cuprate superconductors: stripes, nematics, and superconductivity. Adv. Phys., 2009, 58, P. 699-820.

[20] Vojta M. Stripes and electronic quasiparticles in the pseudogap state of cuprate superconductors. Physica C, 2012, 481, P. 178.

[21] Fujita K. et al. Simultaneous Transitions in Cuprate Momentum-Space Topology and Electronic Symmetry Breaking. Science, 2014, 344, P. 612-616.

[22] Hill R. W. et al. Breakdown of Fermi-liquid theory in a copper-oxide superconductor. Nature, 2001, 414, P. 711-715. 
[23] Smith M.F. et al. Origin of anomalous low-temperature downturns in the thermal conductivity of cuprates. Phys. Rev. B, 2005, 71, P. 014506.

[24] Proust C. et al. Heat transport in $\mathrm{Bi}_{2+x} \mathrm{Sr}_{2-x} \mathrm{CuO}_{6+\delta}$ : Departure from the Wiedemann-Franz law in the vicinity of the metal-insulator transition. Phys. Rev. B, 2005, 72, P. 214511.

[25] Loram J. W. et al. Electronic specific heat of $\mathrm{YBa}_{2} \mathrm{Cu}_{3} \mathrm{O}_{6+x}$ from 1.8 to 300 K. Phys. Rev. Lett., 1993, 71, P. $1740-1743$.

[26] Hawthorn D. G. et al. Field-Induced Thermal Metal-to-Insulator Transition in Underdoped $\mathrm{La}_{2-x} \mathrm{Sr}_{x} \mathrm{CuO}_{4+\delta}$. Phys. Rev. Lett., 2003, 90, P. 197004.

[27] Lalibert F. et al. Origin of the metal-to-insulator crossover in cuprate superconductors. ArXiv e-prints, 2016, arXiv:1606.04491, 16 pp.

[28] Fauque B. et al. Magnetic order in the pseudogap phase of high- $T_{C}$ superconductors. Phys. Rev. Lett., 2006,96, P. 197001.

[29] Li Y et al. Unusual magnetic order in the pseudogap region of the superconductor $\mathrm{HgBa}_{2} \mathrm{CuO}_{4+\delta}$. Nature, 2008,455 , P. 372.

[30] Li Y et al. Magnetic order in the pseudogap phase of $\mathrm{HgBa}_{2} \mathrm{CuO}_{4+\delta}$ studied by spin-polarized neutron diffraction. Phys. Rev. B, 2011, 84, P. 224508.

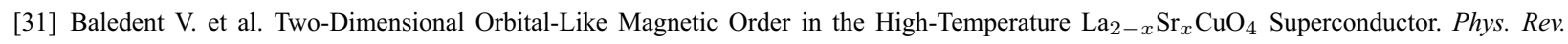
Lett., 2010, 105, P. 027004.

[32] De Almeida-Didry S. et al. Evidence for intra-unit-cell magnetic order in $\mathrm{Bi}_{2} \mathrm{Sr}_{2} \mathrm{CaCu}_{2} \mathrm{O}_{8}+\delta$. Phys. Rev. B, $2012,86, \mathrm{P} .020504$.

[33] Mangin-Thro L. et al. Characterization of the intra-unit-cell magnetic order in $\mathrm{Bi}_{2} \mathrm{Sr}_{2} \mathrm{CaCu}_{2} \mathrm{O}_{8}+\delta$. Phys. Rev. B, 2014, 89, P. 094523.

[34] Norman M. Fermi-surface reconstruction and the origin of high-temperature superconductivity. Physics, 2010,3 , P. 86 (6 pp).

[35] Abdullaev B., Park C. -H. Bosonization of 2D Fermions due to Spin and Statistical Magnetic Field Coupling and Possible Nature of Superconductivity and Pseudogap Phases Below E $E_{g}$.J. Korean Phys. Soc., 2006, 49, P. S642-S646; arxiv:cond-mat/0404668.

[36] Abdullaev B. Implicit Anyon or Single Particle Boson Mechanism of HTCS and Pseudogap Regime. In Trends in Boson Research, edit. by A. V. Ling. N. Y.: Nova Science Publisher Inc., 2006, P. 139-161.

[37] Zavaritsky V. N. et al. Giant normal state magnetoresistances of $\mathrm{Bi}_{2} \mathrm{Sr}_{2} \mathrm{CaCu}_{2} \mathrm{O}_{8+\delta}$. EPJ B, 2004, 42, P. $367-371$.

[38] Mei Jia-Wei et al. Luttinger-volume violating Fermi liquid in the pseudogap phase of the cuprate superconductors. Phys. Rev. B 2012, 85, P. 134519.

[39] Yoshida T. et al. Systematic doping evolution of the underlying Fermi surface of $\mathrm{La}_{2-x} \mathrm{Sr}_{x} \mathrm{CuO}_{4}$. Phys. Rev. B, 2006, 74, P. 224510. 